rungspotenzial. Einerseits werde dadurch die politische Kontrolle gelockert und durch Marktmechanismen ergänzt, was kommerziellen Anbietern den Zugang zu terrestrischen Frequenzen erleichtere. Andererseits könne die Vervielfachung des Angebots zu verstärkter Konkurrenz, sinkenden Werbeeinnahmen und steigenden Produktionskosten führen. Dennoch wird die Digitalisierung nach Ansicht des Autors die grundlegenden Strukturen der Medienlandschaft nicht verändern. Zusätzliche Fernsehanbieter könnten sich je nach regulatorischem Spielraum aber durchaus etablieren.

Als wesentliche inhaltliche Neuerung des digitalen Fernsehens werden gemeinhin interaktive Fernsehformate und Zusatzangebote gehandelt. Dieser Form des Fernsehens widmet sich das fünfte Kapitel. Nach dem Versuch einer Begriffsklärung und Typologisierung interaktiver TV-Dienste gibt Jens F. Jensen einen Überblick über die wichtigsten derzeit verfügbaren Fernsehinhalte dieses Bereichs. Genauer erklärt werden EPG (Electronic Programm Guide), Enhanced TV, Content-on-Demand, Personalised TV, Internet@TV, iTV Werbung, T-Commerce, Spiele, Wetten und Cross Media Interaction.

Am Ende des ersten Teils ergänzt Robert G. Picard die vorwiegend medienpolitische und -ökonomische Perspektive um den Blick auf die Konsumenten. Diskutiert werden weniger die Interessen zukünftiger Nutzer oder mögliche Veränderungen ihres Rezeptionsverhaltens, sondern in erster Linie der Aspekt der Nachfrage als kritischer Faktor für den Erfolg von iTV und DTV. Die Tatsache, dass die Digitalisierung zu Beginn stark von Regierungen und Medienunternehmen ohne Rücksichtnahme auf Konsumentenwünsche vorangetrieben wurde, sieht Picard als ernst zu nehmendes Hindernis für die Implementierung der neuen Technik.

Der zweite, deutlich umfangreichere Teil des Buches beleuchtete Prozesse, Erfahrungen und Resultate jener europäischen Länder, die zum Zeitpunkt des Redaktionsschlusses bereits mit der Einführung von digital-terrestrischem Fernsehen begonnen hatten oder kurz davor standen. Dabei handelt es sich um Großbritannien, Spanien, Schweden, Finnland, Dänemark, Frankreich, Deutschland und Italien. In den acht Fallstudien werden nicht nur Erfolge, sondern auch Schwierigkeiten und Fehlschläge der Frühphase von DTTV erörtert und auf Ursa- chen hin untersucht. Eine Fülle an Hintergrundinformationen ermöglicht dem Leser einen guten Einblick in Zusammenhänge zwischen strukturellen Rahmenbedingungen, Strategien von Medienunternehmen und politischen Entscheidungen der jeweiligen Länder. In der Zusammenschau der Beiträge zeichnen sich darüber hinaus auch allgemeine Mechanismen ab.

Abgeschlossen wird der Band mit einem Resümee der Herausgeber, das noch einmal alle thematischen Stränge zueinander in Verbindung setzt und mit Beispielen aus den Länderberichten untermalt. Zusammenfassend wird deutlich, dass die digitale Technologie der analogen zwar überlegen ist, die vollständige Digitalisierung der Fernsehdistribution für Medienpolitik, Rundfunkindustrie und Konsumenten aber nicht nur Vorteile, sondern auch Risiken, Unsicherheiten und Herausforderungen mit sich bringt.

\section{Christina Ortner}

\section{Werner Wirth / Edmund Lauf / Andreas Fahr (Hrsg.)}

\section{Forschungslogik und -design in der Kom- munikationswissenschaft}

Band 1: Einführung, Problematisierungen und Aspekte der Methodenlogik aus kommunikationswissenschaftlicher Perspektive

Köln: Halem, 2004. - 272 S.

ISBN 3-931606-53-8

Wie die Herausgeber des Buches in ihrer Einleitung zu Recht feststellen, gibt es keine eigenständige Methodologie, durch welche sich die Kommunikationswissenschaft von anderen empirischen Sozialwissenschaften unterscheiden würde. Damit stellt sich die Frage, worin der Sinn einer eigenständigen Publikation über Forschungslogik und -design in der Kommunikationswissenschaft bestehen könnte. Die Antwort der Herausgeber lautet: „Probleme der Forschungslogik [zeigen sich] aus kommunikationswissenschaftlicher Perspektive wenn schon nicht in völlig neuer Form, dann doch in einer spezifischen Gewichtung" (S. 7). Angezielt ist vor diesem Hintergrund eine Auseinandersetzung mit der „Anwendung von Forschungslogik in zentralen Forschungsfeldern der Wirkungs- und Rezeptionsforschung" (S. 8), wobei die „empirische Beweis- und Ar- 
gumentationsstruktur bestimmter Methoden, Designs“ (S. 8) im Mittelpunkt stehen soll. Ein Folgeband beschäftigt sich analog mit „Forschungsansätzen“.

Wenn es einen Bezugspunkt gibt, der allen 12 in diesem Buch versammelten Beiträgen gemeinsam ist, dann handelt es sich um das Konzept der Kausalität. Die ersten beiden Kapitel problematisieren dieses Konstrukt auf grundsätzliche Weise: Früh diskutiert die Unvermeidbarkeit subjektiv-interpretativer Bestandteile von Kausaldeutungen sozialer Phänomene und offeriert aus dynamisch-transaktionaler Sicht Vorschläge, wie mit diesem Problem in der Forschungspraxis umgegangen werden kann. Koschel \& Bilandzic erörtern neuere Entwicklungen in der Diskussion um den Stellenwert kausaler Erklärungen in handlungstheoretischen, am Konzept des Verstehens orientierten Studien. Die restlichen Kapitel befassen sich demgegenüber mit Detailproblemen der Forschungspraxis, mit denen Projekte konfrontiert sind, die vom Standardkonzept der Kausalität ausgehen, wie es beispielsweise in dem Kapitel von Scheufele bestimmt wird - auf die Existenz einer Ursache-Wirkungs-Kette deutet hin, dass zwischen zwei Variablen ein statistischer Zusammenhang besteht, die mutmaßliche Ursache der Wirkung zeitlich vorausgeht und Konfundierung durch Drittvariablen ausgeschlossen ist (S. 245). Einen klaren Schwerpunkt bildet die Experimentalforschung, der alleine fünf Kapitel gewidmet sind. Diese setzen sich mit unterschiedlichen methodischen Herausforderungen von Experimenten auseinander, zu denen teilweise auch nützliche praxeologische Anregungen unterbreitet werden: mit dem Verhältnis von interner und externer Validität (Trepte \& Wirth), der Generalisierungsproblematik bezogen auf die Auswahl der Probanden (Daschmann) und die situativen Kontextbedingungen (Roessing \& Esser), dem Zurechnungsproblem bei mehrdimensionalem Stimulusmaterial (Woelke) sowie den besonderen Herausforderungen, die sich aus den interaktiven Eigenschaften neuer Medien (Internet, Computerspiele) für die experimentelle Wirkungsforschung ergeben (Klimmt, Vorderer \& Ritterfeld). Drei weitere Kapitel thematisieren quasiexperimentelle Alternativen zur genuin experimentellen Forschung: Kolb diskutiert Probleme der international vergleichenden Forschung und konzentriert sich dabei vor allem auf die Frage, wie die funktionale Äquivalenz von Konstrukten, Messinstrumenten und Populationen gesichert werden kann. Scherer und Maurer setzen sich mit Problemen der Erhebung und Analyse von Panelbefragungen auseinander: der oftmals fehlenden Korrespondenz zwischen der Länge der Intervalle zwischen Befragungswellen und der Dauer der Kausalprozesse, die durch die erhobenen Daten abgebildet werden sollen, sowie der Mortalitäts- und Konditionierungsproblematik bei langfristigen Panelbefragungen mit zahlreichen Erhebungswellen. Das Buch schließt mit zwei Kapiteln, welche in nichtformaler Weise die Grundlogiken statistischer Analyseverfahren vorstellen, und zwar von Strukturgleichungsmodellen (Brandl) und Zeitreihenanalysen (Scheufele).

Der Anspruch, diese Gegenstände aus ,einer dezidiert kommunikationswissenschaftlichen Perspektive heraus zu analysieren [und] zu systematisieren" (S. 8), wird nur teilweise eingelöst. Am besten gelingt das einigen Beiträgen, die ihre Problemstellungen anhand ausführlich dargelegter, konkreter kommunikationswissenschaftlicher Projekte diskutieren (z. B. dem Kapitel von Kolb, das auf einer vergleichenden Analyse deutscher und französischer Fernsehnachrichten basiert). Andere Kapitel illustrieren ihre Argumente durch Verweise auf Beispiele aus der kommunikationswissenschaftlichen Forschungsliteratur. Einige können aber eher als allgemeine Beiträge zur sozialwissenschaftlichen Methodendiskussion gelesen werden und lassen allenfalls schwache $\mathrm{Be}-$ züge zu genuin kommunikationswissenschaftlichen Fragestellungen erkennen (etwa die Beiträge von Früh, Koschel \& Bilandzic, Roessing \& Esser, Maurer und Brandl). Insgesamt trägt der Band stark eklektischen Charakter. Ein umfassendes Lehrbuch kann er keinesfalls ersetzen, auch wenn einige Kapitel durchaus als Einführungen zu ihren speziellen Gegenständen nützlich sein mögen. Sein Wert besteht vor allem darin, für eine Reihe von speziellen Problemen, die sich in der Forschungspraxis stellen, zu sensibilisieren und teilweise auch Lösungswege vorzuschlagen.

Rüdiger Schmitt-Beck 